\title{
Central venous catheter-associated bloodstream infections in hemodialysis patients: Another patient safety bundle?
}

\author{
B Lynn Johnston $M D^{1}$, John M Conly $M D^{2}$
}

I $\mathrm{n}$ a previous issue of The Canadian Journal of Infectious Diseases \&8 Medical Microbiology, we reviewed the 'Safer Healthcare Now!' campaign's focus on reducing central venous catheter (CVC)-associated bloodstream infections (BSIs) as a way of improving patient safety (1). This initiative is focused on preventing CVC-associated BSIs in intensive care units. However, other patient groups are also at risk for CVC-related BSIs, suggesting that there are other individuals who would benefit from preventive efforts. A 1996 hospital-wide survey of nosocomial bacteremia in an Israeli university hospital (2) found that $9 \%$ of infected patients were on chronic hemodialysis. Surveillance in 73 hospitals in England between 1997 and 2001 found a CVC BSI rate of 21/1000 nephrology patients at risk who were hospitalized in teaching hospitals (3). This rate was similar to that found in special care neonatal units, although not quite one-half that of patients in a general intensive care unit. In a population-based survey performed in the Calgary Health Region from 2000 to 2002 (4), hemodialysis (HD) posed the greatest risk (RR 208.7; 95\% CI 142.9 to 296.3) for acquiring severe BSI.

The Canadian Organ Replacement Register is the national information system on renal failure and renal dialysis activities in Canada. It reports that between 1993 and 2002, there was a $55 \%$ increase (from 102 patients/million population to 158 patients/million population) in the rate of incident endstage renal disease (ESRD) patients requiring renal replacement therapy (5). This rate is approximately one-half of that reported from the United States (5). Hemodialysis was the predominant renal replacement therapy for new ESRD in 2002 in Canada, with $80 \%$ of these patients started on HD, compared with $63 \%$ in 1993 (5). In terms of absolute numbers, 1822 patients began HD in 1993 and 3945 began this treatment in 2002, for a total of 17,116 patients registered as being on dialysis - a 111\% increase from 1993 (5). A CVC served as the access type for $37 \%$ of patients in 2002 (5). It is clear from these numbers that a great many Canadians are at risk for HD-related BSI and that this number will only increase as the population ages.

The occurrence of bacteremias over a six-month period in chronic HD patients in 19 dialysis units in France was studied prospectively in 1994 (6). Most patients attended hospital dialysis centres (both university and nonuniversity affiliated).
The incidence of bacteremic episodes was $0.93 / 100$ patientmonths and the predominant pathogens were Staphylococcus aureus and coagulase-negative staphylococci (20 and 15 of 51 bacteremias, respectively). Independent risk factors for bacteremia were type of vascular access, history of bacteremia, corpuscular hemoglobin and immunosuppressive therapy. Prospective BSI surveillance in 11 adult Canadian HD units was performed from December 1998 to May 1999, involving $133,158 \mathrm{HD}$ procedures and 316,953 patient-days (7). The BSI rate was found to be $1.4 / 1000$ procedures $(0.6 / 1000$ patientdays), ranging from $0.2 / 1000$ procedures with arteriovenous (AV) fistulae to $3.1 / 1000$ procedures for tunnelled and 5.2/1000 procedures for nontunnelled CVCs. A substudy of patients starting $\mathrm{HD}$ with a new CVC in nine of these units found a somewhat higher rate of BSI at 11.97/10,000 patientdays and 28.81/10,000 dialysis procedures (8). The risk of BSI was significantly higher for patients dialyzed through cuffed (RR 8.49; 95\% CI 3.03 to 23.78) and uncuffed (RR 9.87; 95\% CI 3.46 to 28.20) CVCs as compared with an AV fistula, with no excess risk for dialysis through an AV graft (RR 1.47; 95\% CI 0.36 to 5.96). Other risk factors for BSI, on multivariable analysis, were previous BSI, patient hygiene and superficial access-site infection. $S$ aureus and coagulase-negative staphylococci each accounted for $36 \%$ of the BSIs (7). Investigators in Saudi Arabia had similar findings, with a BSI rate of 3.95/1000 dialysis sessions from August 1999 to January 2001 (9). In this study, rates ranged from 1.29/1000 dialysis sessions for AV fistulae/grafts to 8.30/1000 dialysis sessions for nontunnelled catheters. Finally, the Dialysis Surveillance Network in the United States (US) reported an overall vascular access BSI rate of $1.78 / 100$ patient-months from its 109 participating centres, with rates varying by access type from $0.25 / 100$ patientmonths for AV fistulae to $4.84 / 100$ patient-months for cuffed and 8.73/100 patient-months for noncuffed CVCs (10).

The latter studies point to the role of access type as a major determinant of developing a BSI. Studies generally also point to colonization with $S$ aureus as a risk for HD-related BSI $(6,11)$. The relative roles of other factors such as poor hygiene (8), iron storage levels (12), immunosuppression (6), diabetes mellitus $(11,12)$ and low albumin remain to be defined $(13)$. Two studies $(7,10)$ found that BSI rates varied substantially among dialysis centres, suggesting that being dialyzed at a

${ }^{1}$ Queen Elizabeth II Health Sciences Centre and Dalhousie University, Halifax, Nova Scotia; ${ }^{2}$ Departments of Pathology and Laboratory

Medicine, Medicine, and Microbiology and Infectious Diseases, Centre for Antimicrobial Resistance, University of Calgary, Calgary, Alberta

Correspondence and reprints: Dr B Lynn Johnston, Queen Elizabeth II Health Sciences Centre, 5014ACC - 1278 Tower Road, Halifax,

Nova Scotia B3H 2Y9. Telephone 902-473-5553, fax 902-473-7394, e-mail ljohnsto@dal.ca 
particular centre may increase the risk for an HD-related BSI and that surveillance results may be used for quality improvement.

Information from the US Renal Data System indicated that infection accounted for $15.5 \%$ of deaths in adults with ESRD from 1993 to 1995 (13). In their study of 110 HD patients with CVC BSI, Siegman-Igra et al (2) identified an attributable mortality of $17 \%$. Hemodialysis patients in the US Dialysis Surveillance Network had 14.3 hospitalizations (not confined to infection), 3.82 outpatient intravenous antimicrobial treatment courses and 3.22 vascular access infections (local and BSI) per 100 patient-months (10). A retrospective analysis of data collected on HD patients at one US centre found that 32 of $2239(1.4 \%)$ patients developed infective endocarditis between 1990 and 2000, with a one-year mortality of 56.3\% (14). S aureus accounted for $84 \%$ of infections. This rate was similar to that in another US centre, where eight of $445 \mathrm{HD}$ patients $(1.8 \%)$ developed $S$ aureus endocarditis over an 18-month period (15). Of more concern, however, is that endocarditis complicated $12 \%$ of $S$ aureus BSI in these patients (15). Other complications of S aureus BSI in this latter study included osteomyelitis (11\%), septic arthritis (3\%), epidural abscess (3\%) and death (14\%). In another study (16), these same authors identified $210 \mathrm{HD}$ patients with $S$ aureus BSI from 1994 to 2001. Vascular access was the suspected source of the BSI in $88 \%$ of these patients, with only $1 \%$ of them being dialyzed through an AV fistula. Complications occurred in $31 \%$ of bacteremic patients, most often endocarditis or another metastatic infection. The 12-week mortality was $19 \%$, and the relapse rate was $12.9 \%$. The mean cost of treating $S$ aureus BSI was US\$24,034 per episode. Of interest is a recent study by Ishani et al (17), which showed that septicemia or bacteremia was an independent risk factor for death, myocardial infarction, peripheral vascular disease and stroke in a 1996/1997 cohort of US dialysis patients. Ishani et al speculated that septicemia contributes to the burden of cardiovascular disease in this population through its inflammatory effects.

Given the number of HD-related BSIs that occur, one would anticipate the availability of treatment guidelines based on evidence from randomized, controlled trials. Disappointingly, this is not the case, although there are several studies describing the outcomes associated with treatment strategies employed by its investigators. The main area of debate regarding the management of CVC-related BSI in the $\mathrm{HD}$ patient is related to catheter salvage.

One of the larger studies examining the outcome of CVCrelated bacteremia was conducted at four dialysis centres affiliated with Duke University Medical Center (North Carolina, USA) between April 1995 and January 1996 (18). All patients had tunnelled CVCs. The clinical management of patients, including choice and duration of antibiotics, was left to the discretion of the treating physician. The physicians were, however, provided with guidelines for line removal. Their guidelines recommended prompt CVC removal in patients who developed signs of sepsis, tunnel infection or persistent fever ( $72 \mathrm{~h}$ or longer) or bacteremia during antibiotic therapy. In this study, 41 of 102 patients developed 62 BSIs (3.9/1000 catheter-days), with 63\% caused by Gram-positive cocci. Most of the BSIs (77\%) and 90\% of the Gram-positive BSIs were treated for longer than two weeks. In 24 of 62 (39\%) episodes, the CVC was removed within three days of the BSI. In 26 of $38(68 \%)$ episodes, the CVC was later removed because of infection. Of the 12 catheters successfully salvaged, only six were in place at the end of the three-month follow-up. Nine of the 41 bacteremic patients $(22 \%)$ had complications. Although complications were not more frequent in patients in whom catheter salvage was attempted, the incidence of recurrent bacteremia was higher in this group (RR 4.1; 95\% CI 1.6 to 10.3). This study suggests that while catheter salvage may be successful for some patients, its overall success is limited and may increase the risk for recurrent bacteremia.

In a study by Beathard (19), one of three strategies was used to manage 123 episodes of CVC-related BSI in HD patients. In patients with severe symptoms $(n=37)$, the catheter was removed as soon as practical. Patients with mild symptoms underwent either an over-the-wire change $(n=49)$ or placement of a new line $(n=37)$, dependent on whether there was clinical evidence of tunnel or exit site infection. Culture and clinical cure rates were similar in the three groups, all of whom were treated with antibiotics for 21 days, suggesting to the investigators that it was possible to preserve the catheter in selected patients. A three-pronged strategy, also determined at the discretion of the attending nephrologist, was employed to manage 86 episodes of BSI in 52 patients dialyzed at one university centre from November 1995 to October 1997 (20). At 30-day follow-up, patients treated with antibiotics and overthe-wire exchange experienced higher cure rates than those treated with antibiotics alone $(81.4 \%$ and $36.7 \%$, respectively; $\mathrm{p}=0.0005)$. All patients received antibiotics for 21 days and five patients had immediate catheter removal because of severe sepsis. In an attempt to determine whether over-the-wire exchange and catheter removal with delayed replacement have a similar outcome, Tanriover et al (21) retrospectively compared these two strategies in 69 patients with CVC-associated BSI. Infection-free survival time, mortality and catheter malfunction rates were found to be similar in both groups.

The evidence from these studies supports the need for catheter removal in the face of tunnelled catheter-associated BSI. The question remains whether catheter exchange is as effective as removal and delayed replacement in terms of prolonged infection-free survival. Certainly, catheter exchange avoids the logistical problem of providing dialysis when the access has been removed and minimizes the number of access procedures the patient is exposed to. Investigators have studied the use of antibiotic lock solutions as an adjunct to systemic antimicrobials in treating CVC-associated BSI without catheter removal $(22,23)$. These investigations have been small, noncomparative studies, leaving it impossible to make a recommendation in support of their routine use.

The US Centers for Disease Control and Prevention (CDC) has published guidelines for the prevention of intravascular catheter-related infections (24). Given that the single biggest risk factor for BSI among HD patients is the presence of a CVC, it stands to reason that the most obvious preventive strategy would be to avoid its use. Indeed, one of the CDC recommendations explicitly states that a fistula or graft be used instead of a CVC for permanent dialysis access (24). In a study of vascular access infections at six outpatient HD centres in the western US, Stevenson et al (25) predicted that they would have had a $24 \%$ reduction in BSIs had they followed the National Kidney Foundation's Dialysis Outcomes Quality Initiative recommendation that $40 \%$ of prevalent patients have a native AV fistula. Placement of a native fistula makes good financial and medical sense. In a comprehensive analysis of 
total vascular access costs for new HD patients in Calgary, Alberta, between July 1999 and November 2001, Manns et al (26) determined that patients selected for AV fistula placement incurred the lowest costs during the first year of dialysis. Despite this, there is good evidence of considerable centre-tocentre variation in vascular access type $(7,27)$. Many factors contribute to suboptimal utilization of AV fistulae, not all of which can be avoided. However, one study identified long delays in obtaining access surgery and a high rate of immature fistulae as two major obstacles explaining the low rate of permanent access in their dialysis population (28). Oliver et al (29) at the University of Toronto identified a need for research to determine methods for increasing early referral and timely fistula creation, as well as tools to predict when hemodialysis will be required.

Although a cuffed CVC is recommended if the period of $\mathrm{HD}$ is anticipated to be more than three weeks, this is not based on evidence from randomized, controlled trials specifically in this population (30). In contrast with the nontunnelled CVC literature, the risk of infection with tunnelled HD catheters placed in the femoral vein does not exceed that of catheters placed in the internal jugular, although they have a shorter patency $(31,32)$. The beneficial effect of topical povidone-iodine at the CVC exit site was demonstrated a number of years ago (33). A more recent study (34) found that Polysporin ointment (Pfizer Canada Inc) reduced the rate of infections and improved survival in patients being dialyzed with a cuffed CVC, although this latter finding was not a primary outcome in the study. It has been shown that topical mupirocin is effective in reducing $S$ aureus BSIs in HD patients (35). However, the routine and widespread use of mupirocin has been associated with an increasing prevalence of mupirocinresistant $S$ aureus, making this a less appealing strategy for BSI prevention (36). To avoid this, a group of investigators in Australia looked at the effectiveness of an antibacterial honey in preventing CVC-associated BSI (37). In their study of 101 patients, the incidences of CVC BSIs were comparable in honey - and mupirocin-treated patients (0.97 and 0.85 episodes/1000 CVC-days, respectively). The use of antibacterial honey could hold future promise. As for all CVC insertions, maximal sterile barrier precautions during the procedure are recommended (24).

There has been recent interest in preventing CVC-associated BSIs by instilling an antimicrobial solution into the catheter lumens at the end of each dialysis run. An early study comparing gentamicin $(40 \mathrm{mg} / \mathrm{mL}) /$ citrate with heparin as catheterlock solutions (38) showed longer infection-free catheter survival with gentamicin but reported median predialysis gentamicin levels of $2.8 \mathrm{mg} / \mathrm{L}$, suggesting significant risk for chronic aminoglycoside exposure and ototoxicity. Using a smaller dose of gentamicin (5 mg/mL), McIntyre et al (39) found similar benefits in terms of infection-free survival and random gentamicin levels consistently under $0.2 \mathrm{mg} / \mathrm{L}$. However, depending on the concentration used, gentamicin may be poorly soluble in heparin and perform even more poorly than other antimicrobials in biofilm (40). Given this latter finding, and concerns regarding the emergence of aminoglycoside resistance, other antibiotic lock solutions have been studied. In a randomized study involving $50 \mathrm{HD}$ patients (41), BSI-free survival at 90 days was statistically more likely among patients who received a catheter lock solution containing the antimicrobial agent taurolidine (94\%) than it was among those who received heparin $(47 \%)$. On the other hand, unassisted catheter patency was lower in the taurolidine group (32\% versus $76 \%$; $<0.001$ ). Trisodium citrate (TSC) offers promise as a catheter lock solution with both anticoagulant and antibacterial properties. TSC was compared with heparin in a 17 -month multicentre study (42) that randomly assigned 291 patients into one of two treatment arms: TSC 30\% or heparin. TSC locks were associated with lower CVCassociated BSI rates (1.1 episodes/1000 CVC-days versus 4.1 episodes/1000 CVC-days; $\mathrm{p}<0.001$ ), and the benefit was seen for both tunnelled and nontunnelled catheters. There were fewer major bleeding episodes in the TSC group, and catheter patency was similar between groups. However, systemic instillation of concentrated citrate has the potential to produce life-threatening hypocalcemia (30) and may represent too great a safety concern for HD centres.

It is clear from the literature that CVC-related BSIs are a frequent and costly complication of hemodialysis. As the population ages, it can be expected that the numbers of these infections will only increase. Although more remains to be learned about effective preventive strategies, there are a few well-proven strategies that should be employed. HD-related BSIs as the next patient safety initiative might be a worthwhile target.

\section{REFERENCES}

1. Johnston BL, Conly JM. What do central venous catheter-associated blood stream infections have to do with bundles? Can J Infect Dis Med Microbiol 2005;16:215-8.

2. Siegman-Igra Y, Golan H, Schwartz D, Cahaner Y, De-Mayo G, Orni-Wasserlauf R. Epidemiology of vascular catheter-related bloodstream infections in a large university hospital in Israel. Scand J Infect Dis 2000;32:411-5.

3. Coello R, Charlett A, Ward V, et al. Device-related sources of bacteraemia in English hospitals - opportunities for the prevention of hospital-acquired bacteraemia. J Hosp Infect 2003;53:46-57.

4. Laupland KB, Gregson DB, Zygun DA, Doig CJ, Mortis G, Church DL. Severe blood steam infections: A population-based assessment. Crit Care Med 2004;32:992-7.

5. Canadian Organ Replacement Register. Treatment of End-Stage Organ Failure in Canada, 2002 and 2003. <http://secure.cihi.ca/cihiweb/ dispPage.jsp?cw_page $=$ download_form_e\&cw_sku=0203CORRPDF

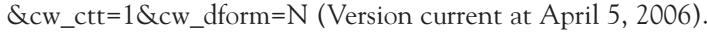

6. Hoen B, Paul-Dauphin A, Hestin D, Kessler M. EPIBACDIAL: A multicenter prospective study of risk factors for bacteremia in chronic hemodialysis patients. J Am Soc Nephrol 1998;9:869-76.

7. Taylor G, Gravel D, Johnston L, Embil J, Holton D, Paton S; Canadian Hospital Epidemiology Committee. Canadian Nosocomial Infection Surveillance Program. Prospective surveillance for primary bloodstream infections occurring in Canadian hemodialysis units. Infect Control Hosp Epidemiol 2002;23:716-20.

8. Taylor G, Gravel D, Johnston L, Embil J, Holton D, Paton S; Canadian Nosocomial Infection Surveillance Program; Canadian Hospital Epidemiology Committee. Incidence of bloodstream infection in multicenter inception cohorts of hemodialysis patients Am J Infect Control 2004;32:155-60.

9. Saeed Abdulrahman I, Al-Mueilo SH, Bokhary HA, Ladipo GO, Al-Rubaish A. A prospective study of hemodialysis access-related bacterial infections. J Infect Chemother 2002;8:242-6.

10. Tokars JI, Miller ER, Stein G. New national surveillance system for hemodialysis-associated infections: Initial results. Am J Infect Control 2002;30:288-95.

11. Saxena AK, Panhotra BR, Venkateshappa CK, et al. The impact of nasal carriage of methicillin-resistant and methicillin-susceptible Staphylococcus aureus (MRSA \& MSSA) on vascular access-related septicemia among patients with type-II diabetes on dialysis. Ren Fail 2002;24:763-77.

12. Teehan GS, Bahdouch D, Ruthazer R, Balakrishnan VS, Snydman DR, Jaber BL. Iron storage indices: Novel predictors of 
bacteremia in hemodialysis patients initiating intravenous iron therapy. Clin Infect Dis 2004;38:1090-4.

13. Nassar GM, Ayus JC. Infectious complications of the hemodialysis access. Kidney Int 2001;60:1-13.

14. Maraj S, Jacobs LE, Kung SC, et al. Epidemiology and outcome of infective endocarditis in hemodialysis patients. Am J Med Sci 2002;324:254-60.

15. Marr KA, Kong L, Fowler VG, et al. Incidence and outcome of Staphylococcus aureus bacteremia in hemodialysis patients. Kidney Int 1998;54:1684-9.

16. Engemann JJ, Friedman JY, Reed SD, et al. Clinical outcomes and costs due to Staphylococcus aureus bacteremia among patients receiving long-term hemodialysis. Infect Control Hosp Epidemiol 2005;26:534-9.

17. Ishani A, Collins AJ, Herzog CA, Foley RN. Septicemia, access and cardiovascular disease in dialysis patients: The USRDS Wave 2 study. Kidney Int 2005;68:311-8.

18. Marr KA, Sexton DJ, Conlon PJ, Corey GR, Schwab SJ, Kirkland KB. Catheter-related bacteremia and outcome of attempted catheter salvage in patients undergoing hemodialysis. Ann Intern Med 1997;127:275-80.

19. Beathard GA. Management of bacteremia associated with tunneledcuffed hemodialysis catheters. J Am Soc Nephrol 1999;10:1045-9.

20. Saad TF. Bacteremia associated with tunneled, cuffed hemodialysis catheters. Am J Kidney Dis 1999;34:1114-24.

21. Tanriover B, Carlton D, Saddekni S, et al. Bacteremia associated with tunneled dialysis catheters: Comparison of two treatment strategies. Kidney Int 2000;57:2151-5.

22. Boorgu R, Dubrow AJ, Levin NW, et al. Adjunctive antibiotic/anticoagulant lock therapy in the treatment of bacteremia associated with the use of a subcutaneously implanted hemodialysis access device. ASAIO J 2000;46:767-70.

23. Krishnasami Z, Carlton D, Bimbo L, et al. Management of hemodialysis catheter-related bacteremia with an adjunctive antibiotic lock solution. Kidney Int 2002;61:1136-42.

24. O'Grady NP, Alexander M, Dellinger EP, et al. Guidelines for the prevention of intravascular catheter-related infections. Clin Infect Dis 2002;35:1281-307.

25. Stevenson KB, Hannah EL, Lowder CA, et al. Epidemiology of hemodialysis vascular access infections from longitudinal infection surveillance data: Predicting the impact of NKF-DOQI clinical practice guidelines for vascular access. Am J Kidney Dis 2002;39:549-55.

26. Manns B, Tonelli M, Yilmaz S, et al. Establishment and maintenance of vascular access in incident hemodialysis patients: A prospective cost analysis. J Am Soc Nephrol 2005;16:201-9.

27. Tokars JI, Light P, Anderson J, et al. A prospective study of vascular access infections at seven outpatient hemodialysis centers. Am J Kidney Dis 2001;37:1232-40.
28. Lee T, Barker J, Allon M. Tunneled catheters in hemodialysis patients: Reasons and subsequent outcomes. Am J Kidney Dis 2005;46:501-8

29. Oliver MJ, Rothwell DM, Fung K, Hux JE, Lok CE. Late creation of vascular access for hemodialysis and increased risk of sepsis. J Am Soc Nephrol 2004;15:1936-42.

30. Allon M. Dialysis catheter-related bacteremia: Treatment and prophylaxis. Am J Kidney Dis 2004;44:779-91.

31. Naumovic RT, Jovanovic DB, Djukanovic LJ. Temporary vascular catheters for hemodialysis: A 3-year prospective study. Int J Artif Organs 2004;27:848-54.

32. Maya ID, Allon M. Outcomes of tunneled femoral hemodialysis catheters: Comparison with internal jugular vein catheters. Kidney Int 2005;68:2886-9.

33. Levin A, Mason AJ, Jindal KK, Fong IW, Goldstein MB. Prevention of hemodialysis subclavian vein catheter infections by topical povidone-iodine. Kidney Int 1991;40:934-8.

34. Lok CE, Stanley KE, Hux JE, et al. Hemodialysis infection prevention with polysporin ointment. J Am Soc Nephrol 2003;13:169-79.

35. Tacconelli E, Carmeli Y, Aizer A, Ferreira G, Foreman MG, D'Agata EM. Mupirocin prophylaxis to prevent Staphylococcus aureus infection in patients undergoing dialysis: A meta-analysis. Clin Infect Dis 2003;37:1629-38.

36. Vivoni AM, Santos KR, de-Oliveira MP, et al. Mupirocin for controlling methicillin-resistant Staphylococcus aureus: Lessons from a decade of use at a university hospital. Infect Control Hosp Epidemiol 2005;26:662-7.

37. Johnson DW, van Eps C, Mudge DW, et al. Randomized, controlled trial of topical exit-site application of honey (Medihoney) versus mupirocin for the prevention of catheter-associated infections in hemodialysis patients. J Am Soc Nephrol 2005;16:1456-62.

38. Dogra GK, Herson H, Hutchison B, et al. Prevention of tunneled hemodialysis catheter-related infections using catheter-restricted filling with gentamicin and citrate: A randomized controlled study. J Am Soc Nephrol 2002;13:2133-9.

39. McIntyre CW, Hulme LJ, Taal M, Fluck RJ. Locking of tunneled hemodialysis catheters with gentamicin and heparin. Kidney Int 2004;66:801-5

40. Droste JC, Jeraj HA, MacDonald A, Farrington K. Stability and in vitro efficacy of antibiotic-heparin lock solutions potentially useful for treatment of central venous catheter-related sepsis. J Antimicrob Chemother 2003;51:849-55.

41. Allon M. Prophylaxis against dialysis catheter-related bacteremia with a novel antimicrobial lock solution. Clin Infect Dis 2003;36:1539-44.

42. Weijmer MC, van den Dorpel MA, Van de Ven PJG, et al; CITRATE Study Group. Randomized, clinical trial comparison of trisodium citrate $30 \%$ and heparin as catheter-locking solution in hemodialysis patients. J Am Soc Nephrol 2005;16:2769-77. 


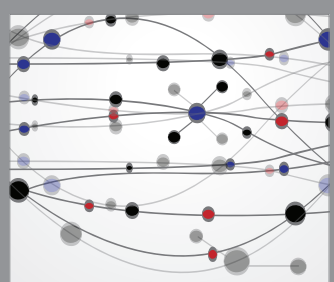

The Scientific World Journal
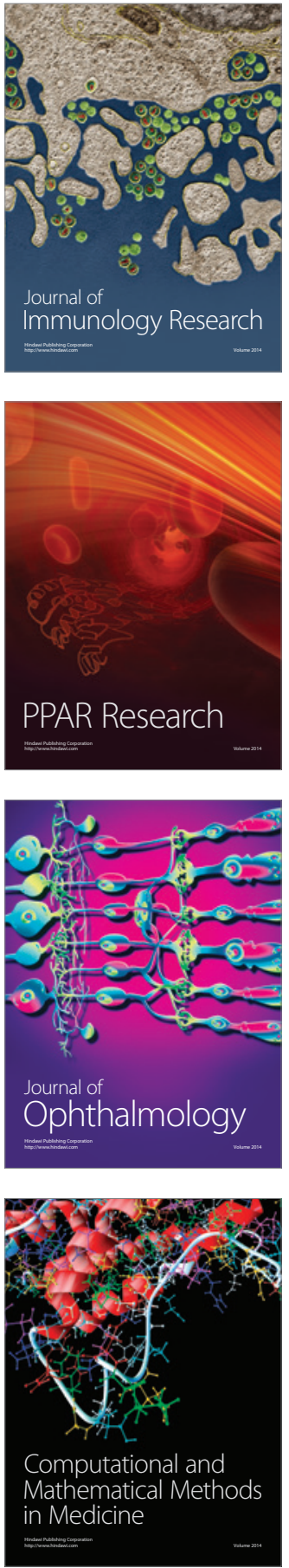

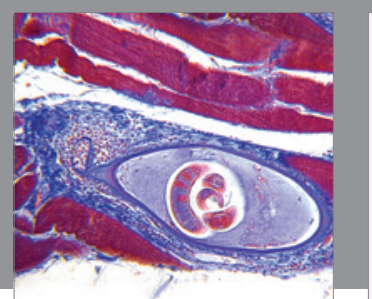

Gastroenterology Research and Practice

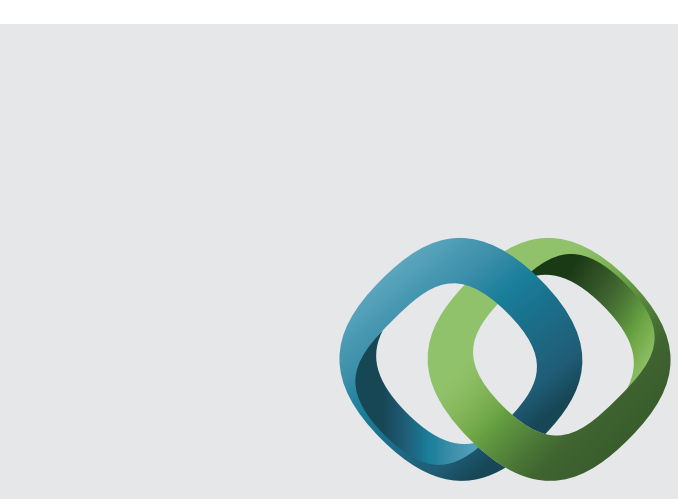

\section{Hindawi}

Submit your manuscripts at

http://www.hindawi.com
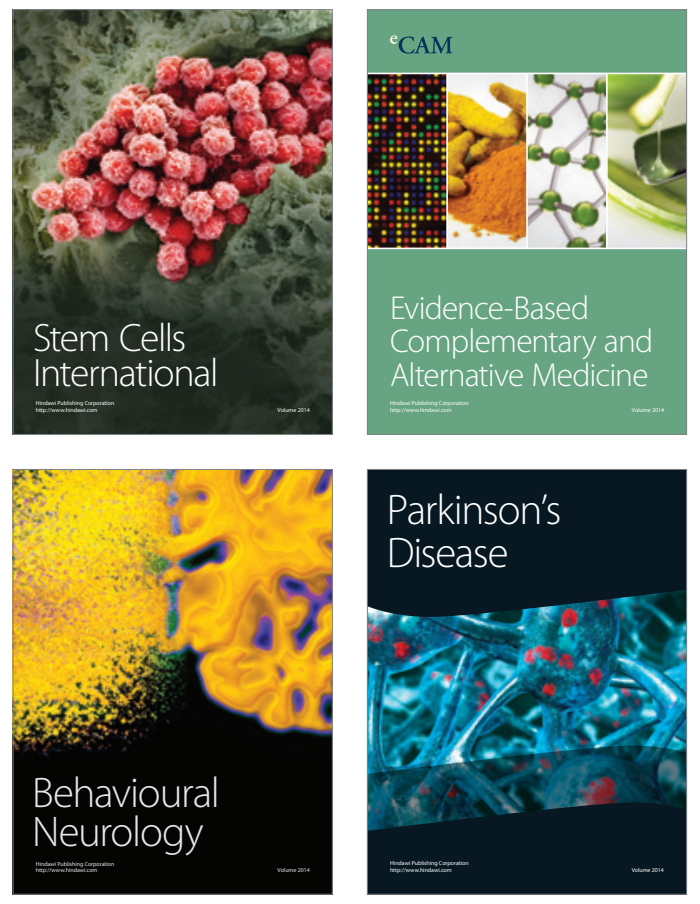
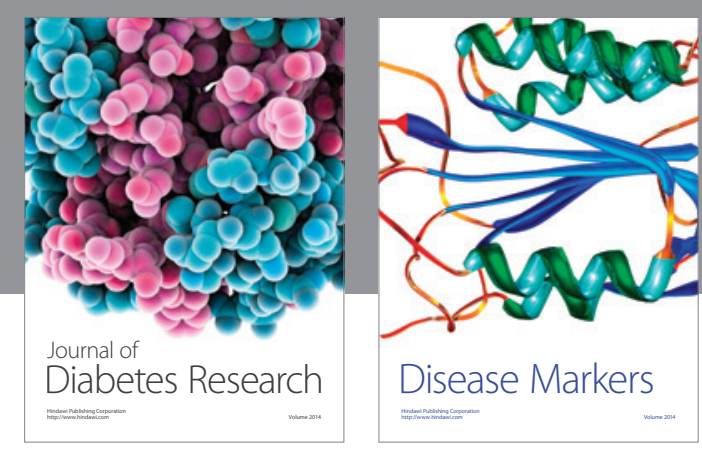

Disease Markers
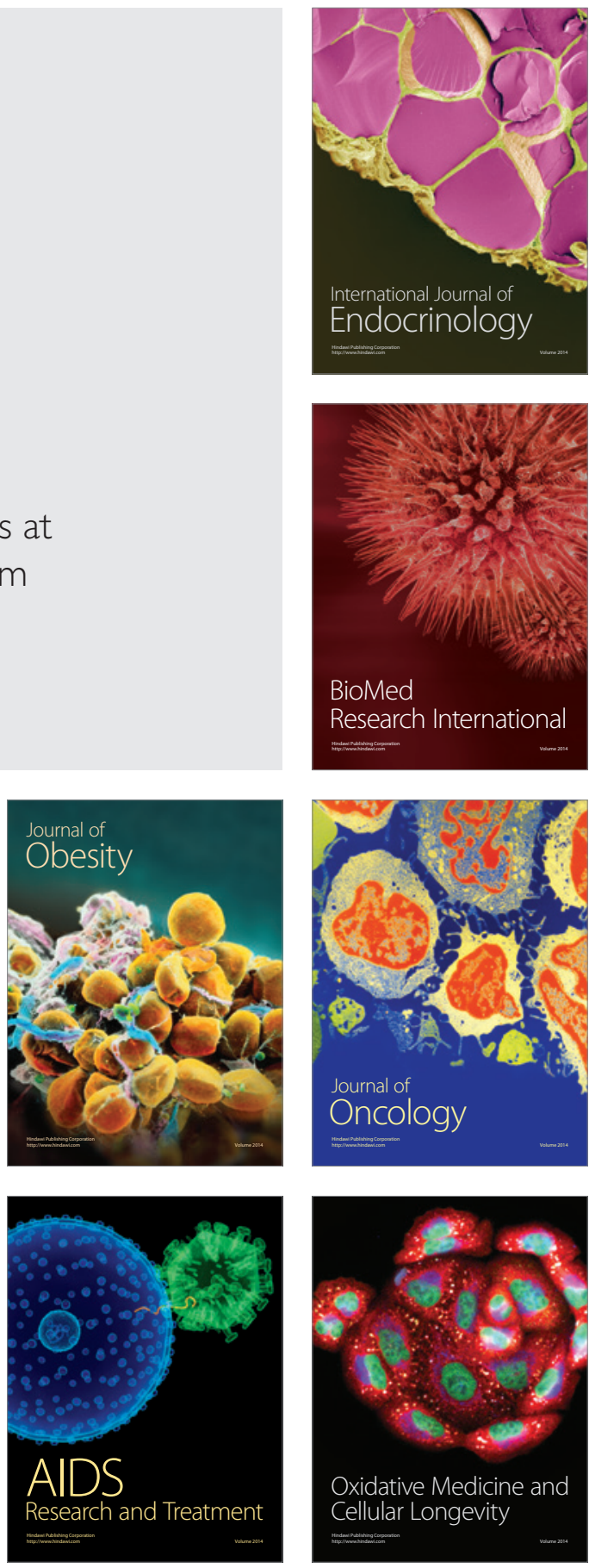